\title{
Project and Realization of a Wide-Range High-Frequency RFID Gate Allowing Omnidirectional Detection of Transponders
}

\author{
Giuliano Benelli, Stefano Parrino, and Alessandro Pozzebon \\ Department of Information Engineering, University of Siena, Via Roma 56, 53100 Siena, Italy \\ Correspondence should be addressed to Alessandro Pozzebon, alessandro.pozzebon@unisi.it \\ Received 6 September 2011; Accepted 17 October 2011 \\ Academic Editor: M. S. Hwang
}

Copyright ( $\odot 2012$ Giuliano Benelli et al. This is an open access article distributed under the Creative Commons Attribution License, which permits unrestricted use, distribution, and reproduction in any medium, provided the original work is properly cited.

We describe the study and development of a 2-meter-wide HF RFID gate providing omnidirectional detection of transponders. Common commercial HF RFID gate structures provide a maximum reading range around $150 \mathrm{~cm}$. Moreover, this value is in most cases guaranteed only for the maximum coupling direction, with lower values for the other 2 orientations. The proposed structure raises the value of the reading range up to $200 \mathrm{~cm}$ for every orientation of the transponder, with even better results $(220 \mathrm{~cm})$ when the transponder is in the position of maximum coupling. This result has been achieved through numerical simulations, focused on the study of the geometry of the antenna system and on the realization of the matching circuit and then confirmed with the physical implementation of the system.

\section{Introduction}

With the term RFID (Radio Frequency Identification) are indicated all the technologies that allow the contactless identification (i.e., the unambiguous recognition) of an item, an animal, or a person using the electromagnetic fields as the mean of communication $[1,2]$.

Obviously the term RFID encompasses several different technological devices, with different operating frequencies and different electronic features. Due to this fact, every time that RFID is chosen as the right technology to be used for a particular application, specific studies have to be done in order to identify the adequate technical solution offering the best ratio among costs and performances.

In addition, the use of electromagnetic fields in particular environments such as hospitals or any other public building can have several limitations due to the presence of people and the interaction with other electronic devices.

While the historical applicative fields of RFID have always been the access control, the industrial tracking, and the surveillance systems, in the last years new scenarios are emerging: one of the most significant is, for example, the field of the Healthcare. The integration of automatic identification techniques with the technological frameworks of hospitals, emergency rooms, or nursing homes can increase the efficiency of all the assistance operations improving the mobility and the accessibility to the informative systems.

The paper is organized as follows. In Section 2, the basic concepts concerning the antennas for HF RFID systems are described, focusing on the materials to be used, on the factors influencing the final performances, and on the matching of the antenna. Section 3 describes the possible configuration of antenna gate structures. In Section 4, the simulations necessary to project a standard gate structure are described, while in Section 5 the simulations carried on to develop the wide-range structure are analyzed. Section 6 describes the physical implementation of the gate structure emerged from the simulations. Finally, Section 7 provides some concluding remarks and future works.

\section{Antennas for HF RFID Systems}

An RFID system is basically composed by the transponders, or tags, which are the smart devices located on the item to be identified, and the reader, which performs the effective identification operations. The project of the antenna structure of the reader is especially important because it can notably 
affect the performances of the system and its installation in the final destination [3-8].

In general, the reading distance of a tag depends mainly on the physical dimensions of the antenna system adopted, because the intensity of the magnetic field increases with the growth of these dimensions.

On the other hand, if the dimensions come through a certain limit the following problems can occur:

(i) Signal-noise ratio (SNR) decreases;

(ii) law values of electromagnetic emissions can be surpassed;

(iii) "holes" can be generated in the emitted magnetic field, creating shade zones where the tag cannot be read;

(iv) the inductance can grow up to a level that makes the matching of the system extremely difficult if not totally impossible.

2.1. The Materials and the Design. An antenna can be ideally built with every kind of conductive material, but in practice the most common solutions are based on the use of

(i) hollow copper tubes (also aluminium is a good option, but is less malleable), with the diameter going from $1 / 2$ inch (i.e., $15 \mathrm{~mm}$ ) for smaller loops (e.g., $500 \mathrm{~mm} * 500 \mathrm{~mm}$ ) up to $3 / 4$ inch (i.e., $22 \mathrm{~mm}$ ) for bigger loops;

(ii) copper strips usually from $10 \mathrm{~mm}$ to $50 \mathrm{~mm}$ wide.

During the realization of an antenna or a system of more antennas, many factors can influence the final performances. Among the most significant the following can be listed.

(i) For the solutions described before the following rule applies: the bigger the antenna is, the wider the diameter of the tube or the width of the strip should be. This fact reduces the inductance of the antenna; a good alternative can be offered by the interconnection of two parallel antennas, in order to obtain the same inductance of a single antenna.

With larger antennas wider reading ranges are achievable, but there is a limit due to the equivalent inductance of the antenna: over $5 \mu$ Henry the matching of the antenna becomes virtually impossible.

(ii) The presence of metal in the proximities of the system can reduce the performances of the antennas. In particular the most part of the negative effect simply derives from the detuning of the antenna and can be resolved readapting the matching circuit. Anyway if the metal is too close it can absorb a great part of the emitted power: for this fact the tuning of an antenna should be made only when the antenna is positioned in its final destination.

(iii) The presence of other antennas next to the system can alter the performances due to the mutual coupling phenomenon: anyway in some cases, for example, in the case of the studied system, this effect is deliberately wanted because it can increase the performances. This fact will be specifically examined in the next sections.

(iv) The activation of the transponders depends directly on the power transmitted from the antenna through the magnetic coupling, but the energy that they can receive is the highest only when they are in the position of maximum coupling, that is, when the antenna loop is parallel to the transponder and the lines of the generated magnetic field are perpendicular to the transponder.

Due to this fact only in this position the reading distance is maximum: when the corner among the transponder and the lines of the field is less than $90^{\circ}$ the coupling decreases lowering the read distance.

2.2. The Matching of the Antenna. Antennas used for inductively coupled passive RFID systems are designed as loops, allowing the magnetic field component in close field conditions, which is the one that enables the reading of the tag, to be prevalent over the electric field component; therefore the input impedance of a loop is essentially reactive (in particular inductive) [9].

The antenna is linked to the reader through a coaxial cable with a characteristic impedance of $50 \Omega$ and it is evident that with a direct link the most part of the input power would be reflected due to the mismatching.

For this fact the antenna too should have a $50 \Omega$ characteristic impedance, and this can be done adding a matching circuit between the coaxial cable and the antenna [10].

Among the various matching techniques the most important are the Gamma matching, the T-matching, the use of balun, and the Capacitive matching. We will consider this last option even if it is perhaps the most complex because small variations of the capacity (in the order of picoFarad) can make a great difference in the matching operation.

The idea is to use a parallel capacitive RC matching network, in order to make the LC parallel circuit resound and to match the real part of the obtained impedance.

This configuration is quite simple to be realized and the values of the components can be adjusted in order to obtain the best matching possible.

The circuit is divided into two parts: the first one, directly linked to the terminals of the antenna, is formed by a resonant circuit at the frequency $f_{0}=13.56 \mathrm{MHz}$. This is composed by the loop inductance, a parallel capacity, and a resistance in order to define the quality factor $Q$ of the circuit.

The second part of the matching circuit is composed by a network which has the task to match the input impedance, now exclusively real, seen at the terminals of the matching resonance circuit.

\section{Antenna Configurations}

3.1. Gate Configurations. One of the most common technical solutions for the tracking operations is the realization of gate structures: in this kind of solutions more antennas, connected to a single reader, create a sort of "Tunnel" through 


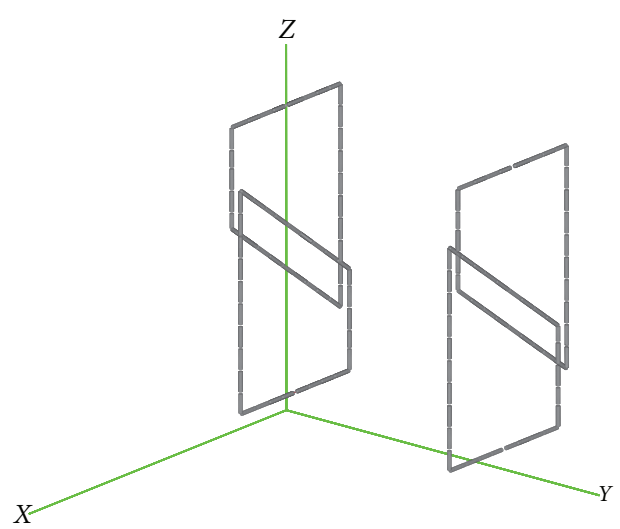

(a)

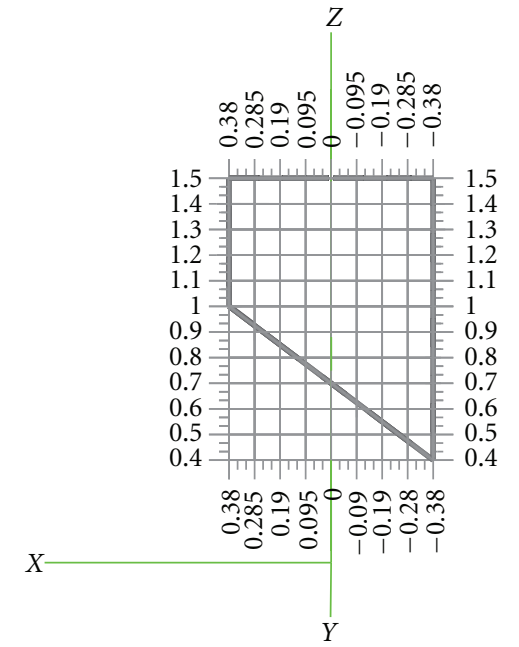

(b)

Figure 1: Gate geometry (a); a single antenna (b).

which the tagged items are carried and at the same time recognized.

Gates can be single, double, or multiple and they can be composed by up to 8 antennas.

Typical applications include solutions integrated in conveyor belts, structures located at the doors of warehouses or at the exits of shops to check the items bought by customers.

The simplest configuration foresees the use of two antennas, typically rectangular, located one in front of the other and linked to a reader through a multiplexer: obviously this kind of configuration covers an overall area approximately twice the one covered by a single antenna.

Two different cases can be studied. In the first one only one antenna is powered, while the second one is only matched and located in front of the other: in this case the performances of the powered antenna increase due to the phenomenon of mutual coupling.

In the second case the both antennas are connected through a "Splitter", and both are powered: in this situation the reading distance can be even more than doubled, due to the fact that each antenna emits its own magnetic field. Anyway a phenomenon of field cancellation can occur in this case deriving from the perspective and the distance of the antennas.

\section{Project and Realization of a Standard Gate}

4.1. Standard Gate Simulations. The first simulations have been carried out on a $1.30 \mathrm{~m}$ wide gate structure: this width represents a good starting point because it is the width covered by common RFID gates.

The proposed geometry is shown in Figure 1(a) and foresees the use of two overlapped antennas on both sides of the gate.

Due to the phenomenon of mutual inductance, the matching of the antenna is different from the case of a single powered antenna and the case of the 4 powered antennas, and an adequate model has been realized [11].

In the first case we have a single powered antenna with the other three put at open circuit and described as mutually coupled inductances with coupling coefficient equal to

$$
K_{i, j}=\frac{M_{i, j}}{\sqrt{L_{i} L_{j}}} .
$$

With four identical antennas we have

$$
\begin{gathered}
L_{i}=L_{j} \Longrightarrow L_{1}=L_{2}=L_{3}=L_{4}=L, \\
K_{i, j}=K_{j, i}=\left.\left(\frac{M_{i, j}}{\sqrt{L_{i} L_{j}}}\right)\right|_{L_{i}=L_{j}=L}=\left(\frac{M_{i, j}}{L}\right) .
\end{gathered}
$$

This representation is justified by the fact that when we calculate $M_{1,2}$ in practice we consider a two-port network where each port is powered by a current generator, and we calculate its $Z$ parameters

$$
\begin{aligned}
& V_{1}=Z_{11} \cdot I_{1}+Z_{12} \cdot I_{2} \Longrightarrow V_{1}=j \omega L \cdot I_{1}+j \omega M_{12} \cdot I_{2}, \\
& V_{2}=Z_{21} \cdot I_{1}+Z_{22} \cdot I_{2} \Longrightarrow V_{2}=j \omega M_{21} \cdot I_{1}+j \omega L \cdot I_{2} .
\end{aligned}
$$

With both the ports powered with the same current $I=I_{1}=$ $I_{2}$ from the first equation we have $V_{1}=j \omega L \cdot I_{1}+j \omega M_{12} \cdot I_{1}$ and we calculate the input impedance

$$
L_{\mathrm{tot}}^{\prime}=L+M_{21}
$$

and then

$$
M_{21}=L_{\mathrm{tot}}^{\prime}-L,
$$

where $L_{\text {tot }}^{\prime}$ is the inductance of the system when the antennas 1 and 2 are powered with the same current while the other 


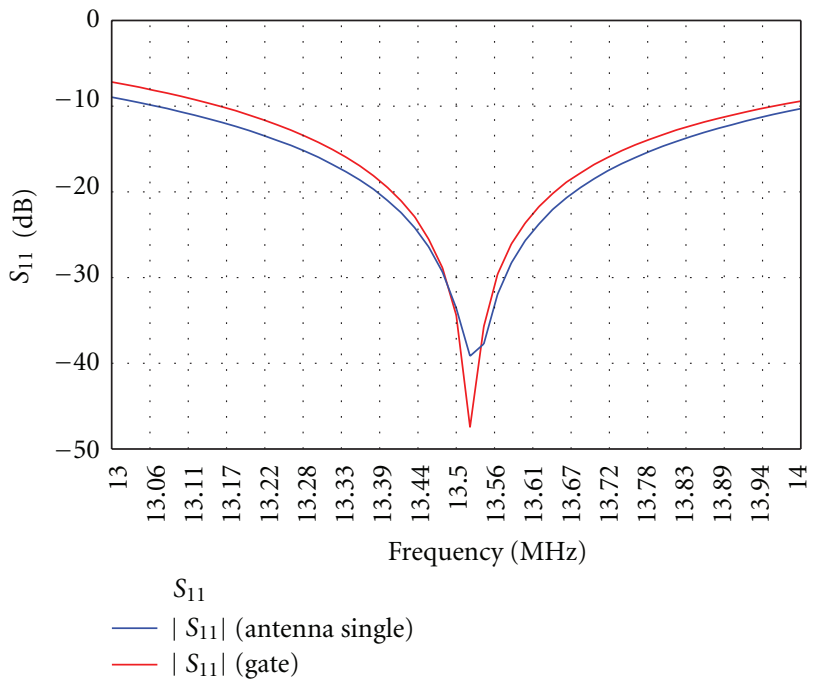

(a)

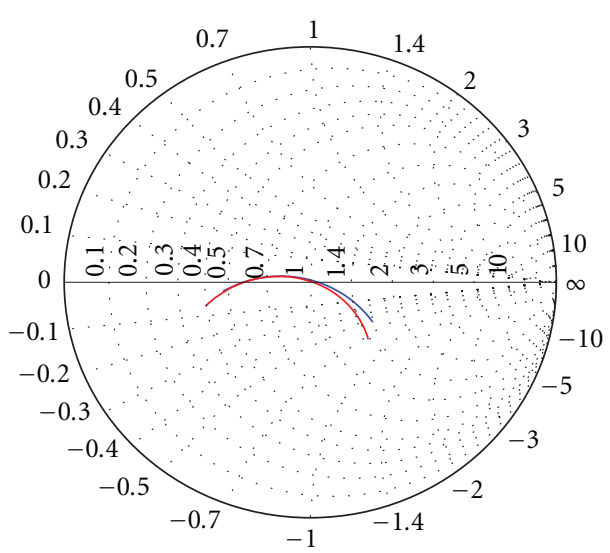

$S_{11}$

- $\left|S_{11}\right|$ (antenna single)

$-\left|S_{11}\right|$ (gate)

(b)

Figure 2: Reflection coefficient on the cartesian graphic (a) and on the Smith Chart (b).

two are put at open circuit, and $L$ is the inductance of a single loop when the other three are put at open circuit. Simulating the system with POSTFEKO and calling 1 and 2 the antennas on one side and 3 and 4 the other two we calculated then the following parameters:

(i) $L$, with only one antenna powered and the others put at open circuit: $\omega L=222.1 \Omega \Rightarrow L=$ $222.1 \Omega /\left.2 \pi f\right|_{f=13.56 \mathrm{MHz}} \simeq 2.608 \mu \mathrm{H}=2608 \mathrm{nH}$;

(ii) $M_{12}$ with only antennas 1 and 2 powered: $\omega L_{\text {tot }}^{\prime}=$ $252.67 \Omega \Rightarrow M_{12}=L_{\text {tot }}^{\prime}-L=252.67 \Omega / \omega-$ $2608 \mathrm{nH} \simeq 358 \mathrm{nH}$;

(iii) $M_{13}$ with only antennas 1 and 3 powered: $\omega L_{\text {tot }}^{\prime}=$ $224.7 \Omega \Rightarrow M_{13}=L_{\text {tot }}^{\prime}-L=224.7 \Omega / \omega-2608 \mathrm{nH} \simeq$ $30.53 \mathrm{nH}$;

(iv) $M_{14}$ with only antennas 1 and 4 powered: $\omega L_{\text {tot }}^{\prime}=$ $224.0377 \Omega \Rightarrow M_{14}=L_{\text {tot }}^{\prime}-L=224.037 \Omega / \omega-$ $2608 \mathrm{nH} \simeq 22.74 \mathrm{nH}$;

(v) $M_{23}$ with only antennas 2 and 3 powered: $\omega L_{\text {tot }}^{\prime}=$ $224.039 \Omega \Rightarrow M_{23}=L_{\text {tot }}^{\prime}-L=224.039 \Omega / \omega-$ $2608 \mathrm{nH} \simeq 25.1 \mathrm{nH}$;

(vi) $M_{24}$ with only antennas 2 and 4 powered: $\omega L_{\text {tot }}^{\prime}=$ $224.99 \Omega \Rightarrow M_{24}=L_{\text {tot }}^{\prime}-L=224.99 \Omega / \omega-$ $2608 \mathrm{nH} \simeq 33.93 \mathrm{nH}$;

(vii) $M_{34}$ with only antennas 3 and 4 powered: $\omega L_{\text {tot }}^{\prime}=$ $252.92 \Omega \Rightarrow M_{34}=L_{\text {tot }}^{\prime}-L=252.92 \Omega / \omega-$ $2608 \mathrm{nH} \simeq 361.97 \mathrm{nH}$.

Analyzing these coefficients we can see that $M_{12}$ and $M_{34}$ present the highest values (ten times higher than the others), which is obvious because this is the mutual induction among the overlapped antennas. Moreover we can see that the values of the couples $M_{13}-M_{24}, M_{14}-M_{23}$ and $M_{12}-M_{34}$ are very similar and in fact they should be identical due to symmetry reasons: they are a bit different due to numerical approximations. Finally we can see that $M_{13}$ and $M_{24}$ are slightly higher than $M_{14}$ and $M_{23}$ because antenna couples $1-3$ and 2-4 are faced and the concatenated magnetic flux is greater.

It is interesting to see the differences in the performances from the case of a single antenna to the case of the gate. From Figure 2 we can see that both the antenna and the gate have a good matching, even if slightly moved $(40 \mathrm{kHz})$ from the operative frequency, but in the case of the gate the band is evidently wider.

4.2. The Magnetic Field. Figure 3(a) shows the representation of the near field $\left|H_{y}\right|$ on the $y$ - $z$ plan with $x=0$, and we can see that the gate presents along the $y$ direction a higher reading distance than the single antenna on the maximum coupling direction of the transponder. The $z$ coordinate has been chosen in order to maximize the field configuration for both the structures, and this is verified approximately at the center of the loop ( $x=0, z=1.10 \mathrm{~m}$ and $y$ variable) for a single antenna and at the center of the overlapping area for the gate ( $x=0, z=0.9 \mathrm{~m}$ and $y$ variable).

A more accurate analysis shows that for $x=0$ the gates has $\left|H_{y}\right| \geq 60 \mathrm{~mA} / \mathrm{m}$ (the minimum value for the tag reading) in the $0.10 \mathrm{~m} \leq$ for all $z \leq 1.5 \mathrm{~m}$ range (the total height of the gate) and for all $y \in[0,1.3 \mathrm{~m}]$ (the distance from the two sides of the gate). The single antenna presents instead $\left|H_{y}\right| \geq 60 \mathrm{~mA} / \mathrm{m}$ in the best case, with $x=0$ and $z=1.10$ in the $0 \mathrm{~m} \leq$ for all $y \leq 1.09 \mathrm{~m}$ range, and in the worst case, with $x=0$ and $z=0.4$ in the $0 \mathrm{~m} \leq$ for all $y \leq 0.25 \mathrm{~m}$ range.

Figure 3(b), 3(c) show $\left|H_{x}\right|$ along $y$ at the variation of $z$, respectively, for the gate and for the single antenna: in the first case we can see that in the best case $(z=0.7 \mathrm{~m})\left|H_{x}\right| \geq$ $60 \mathrm{~mA} / \mathrm{m}$ up to $y \simeq 0.47 \mathrm{~m}$ while with the single antenna, in the best case $(z=0.6 \mathrm{~m})$, we reach the value of $y \simeq 0.37 \mathrm{~m}$. 

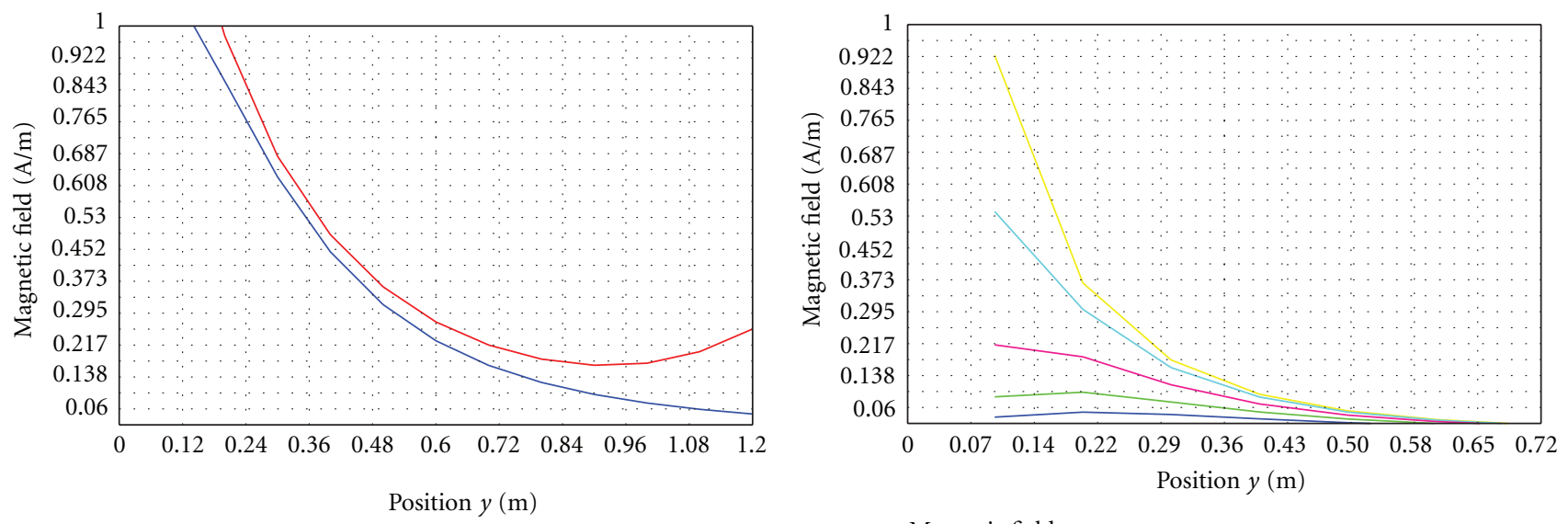

Magnetic field

— Near Field $\left|H_{y}\right|$ (gate)

— Near Field $\left|H_{y}\right|$ (antenna single)

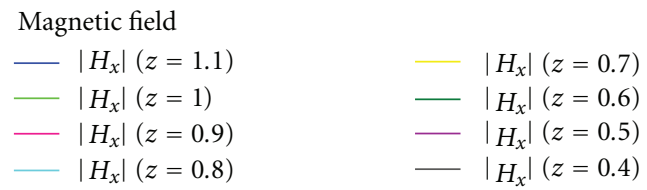

(a)

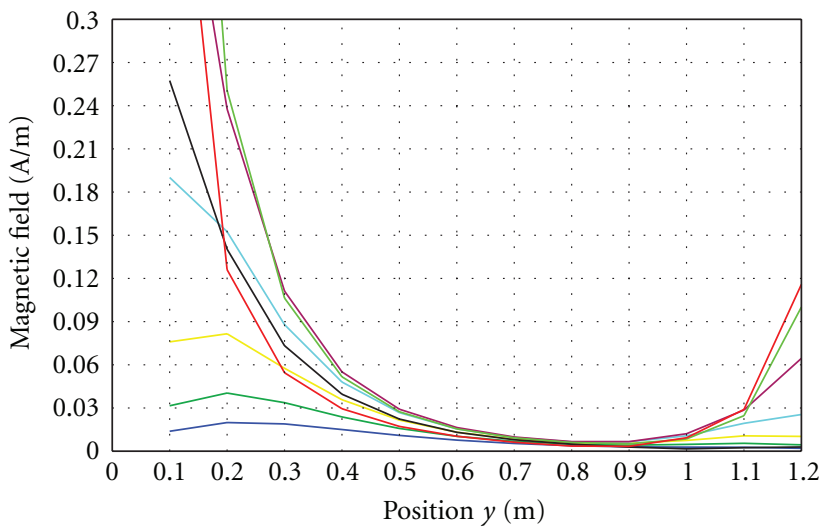

(b)

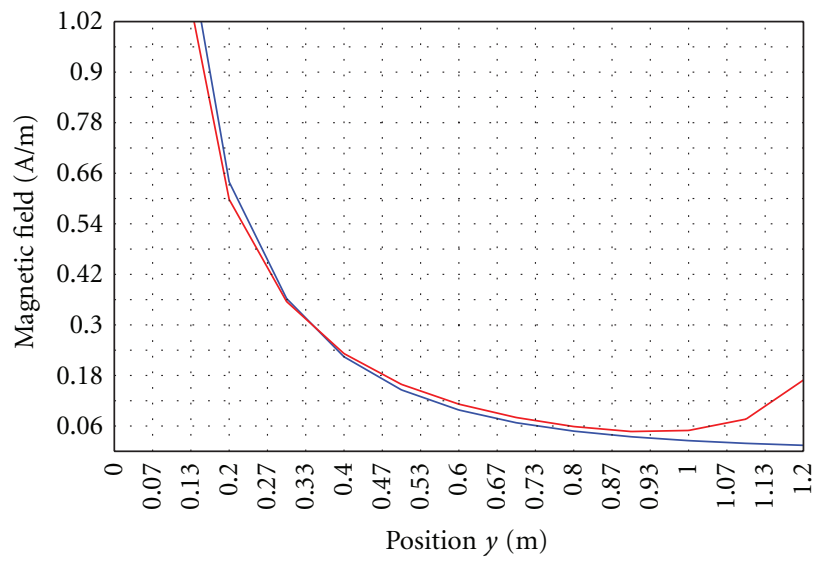

Magnetic field

$$
\begin{array}{rr}
-\left|H_{x}\right|(z=0.9) & -\left|H_{x}\right|(z=0.5) \\
-\left|H_{x}\right|(z=0.8) & \left|H_{x}\right|(z=0.4) \\
-\left|H_{x}\right|(z=0.7) & -\left|H_{x}\right|(z=0.3) \\
-\left|H_{x}\right|(z=0.6) & -\left|H_{x}\right|(z=0.2)
\end{array}
$$

Magnetic field

$-\left|H_{z}\right|(z=1.5$ gate $)$
— Near field $\left|H_{z}\right|(z=1.5$ antenna single $)$

(d)

(c)

Figure 3: $\left|H_{y}\right|$ on the $y$ - $z$ plain with $x=0$ for the single antenna (blue) and the gate (red) (a); $\left|H_{x}\right|$ along $y$ at the variation of $z$ for the gate (b) the single antenna (c); $\left|H_{z}\right|$ for $x=0, y$ variable and $z=1.5 \mathrm{~m}$ for the single antenna (blue) and the gate (red) (d).

Finally Figure 3(d) shows the values of $\left|H_{z}\right|$ for $x=0, y$ variable and $z=1.5 \mathrm{~m}$ (the value at which both the antenna and the gate present maximum $\left.\left|H_{z}\right|\right)$. While in the case of the gate, performances seem to be worse than in the case of the single antenna, we can notice the rise of an interesting phenomenon: first of all, due to mutual coupling, an induced magnetic field is created also in correspondence to the not powered antenna for $x=0$; next to this, two field lobes appear exactly in correspondence to the overlapping, where mutual coupling plays an important role; finally we can notice an increase of the field also in correspondence to the overlapping area of the two facing antennas, underlining also in this case the presence of a remarkable mutual induction.

Moreover we also have to consider that the single antenna presents higher values first of all thanks to the limited dimensions of its geometry, and then because the regions where the tag is detectable are thinner than the ones provided by the gate.

This is also argued by the fact that, with the same powering, the current flowing through the powered antenna of the gate is lower than the current flowing through the single antenna, even if the performances are higher. 


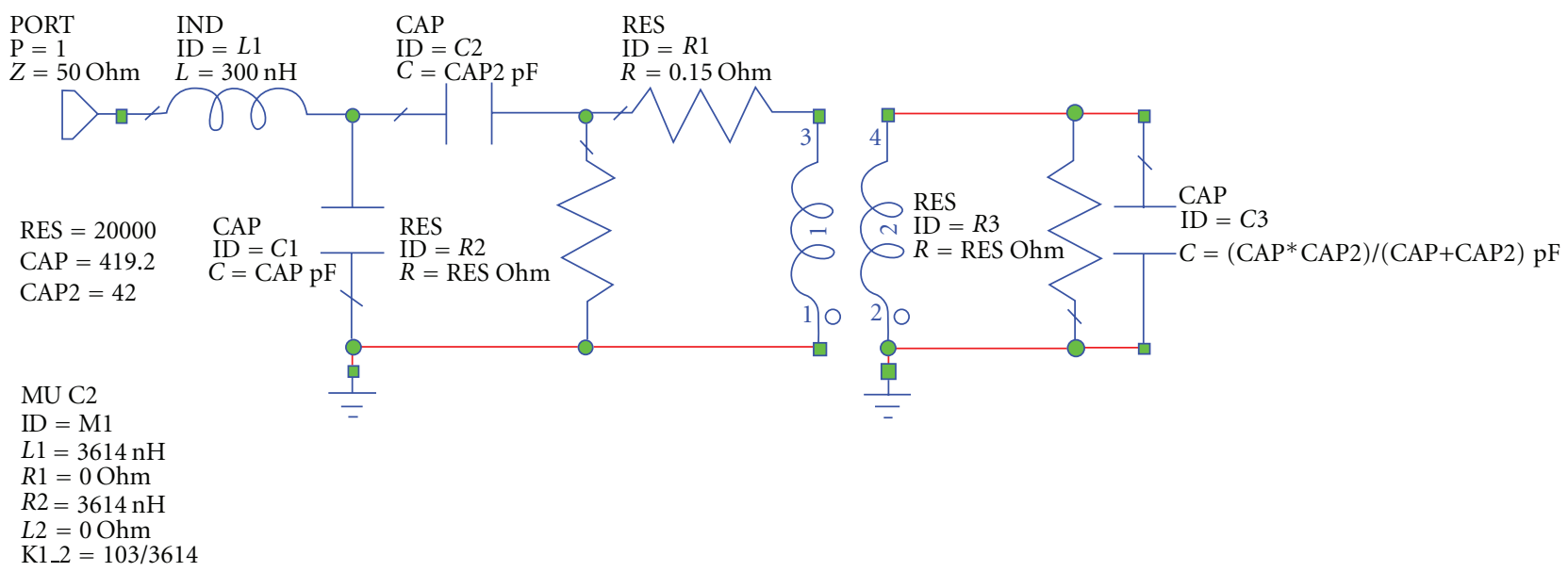

FIgURE 4: Matching circuit with AWR.

\section{Project of the Wide-Range Gate: Simulations}

5.1. The Geometry. The first simulations have focused on the search of the right geometry: at the end of the study the width of overlapping surface has been fixed at $22 \mathrm{~cm}$. This has proved to be the best result because on equal current flow the generated field is the highest in the three dimensions.

The four antennas obviously present the same dimensions and their perimeter is equal to $(55+90+145+$ $90 \sqrt{2}) \mathrm{cm} \simeq 4.173 \mathrm{~m}$; in our case wavelength is equal to $\lambda=$ $c / f=22.12 \mathrm{~m}$ : we can then affirm that every loop is around $\lambda / 5$ wide. The system composed by two overlapped antennas is $1.78 \mathrm{~m}$ high and $0.90 \mathrm{~m}$ wide. In the final disposition the system will be raised $12 \mathrm{~cm}$ above the floor: if we consider the system as placed on the $x-z$ plan we have $0.12 \mathrm{~m} \leq z \leq$ $1.90 \mathrm{~m}, x=0$ and $y=0$ (while for the other half of the gate we have $y=2 \mathrm{~m}$ ), with the center of the system located at $z \simeq 1 \mathrm{~m}$.

The geometry has been realized with CADFEKO simulation environment, while with POSTFEKO we calculated the values of the impedance of each loop and the mutual inductance between the overlapped antennas:

$$
\begin{aligned}
& \left.\left(\omega L_{1}\right)\right|_{f=13.56 \mathrm{MHz}} \\
& =307.76 \Omega \Longrightarrow L_{1}=L_{2}=L_{3}=L_{4}=3614 \mathrm{nH}, \\
& \omega L_{12}=L_{21}=316.53 \Omega \text {, } \\
& M_{12}=M_{21}=\frac{\omega L_{12}-\omega L_{1}}{\omega}=103 \mathrm{nH} \text {. }
\end{aligned}
$$

We can finally calculate the quality factor of the antenna $Q=$ $20000 / 316.53 \simeq 60$.

5.2. The Matching. The calculated values have been introduced in the AWR circuit simulator: the matching circuit is shown in Figure 4; the reflection coefficient (Figure 5(a)) is extremely interesting because the cartesian graphic presents two resonance peaks, on the left $(13.48 \mathrm{MHz})$ and on the right $(13.62 \mathrm{MHz})$ of the operative frequency $(13.56 \mathrm{MHz})$ : the coupling phenomenon is used to enlarge the band using the two close resonances.

The values of CAP, CAP2, and RES provided by AWR have been introduced in POSTFEKO: the resulting matching is shown in Figures 5(c) and 5(d).

5.3. The Generated Field. In this section we analyze the configuration of the magnetic field generated by the system, assuming only the upper antenna to be powered.

(i) $\left|H_{x}\right|$ : the $H_{x}$ field presents two lobes in correspondence to the vertical segments of the system: in particular (considering a $x-z$ plan with $y=1 \mathrm{~m}$ ) we have a maximum of the field for the negative $x$ at $x=-0.56 \mathrm{~m}$, where $H_{x}>60 \mathrm{~mA} / \mathrm{m}$ in the range $0.076 \mathrm{~m} \leq$ for all $z \leq 1.22 \mathrm{~m}$, and another one for the positive $x$ at $x=0.64 \mathrm{~m}$, where $H_{x}>60 \mathrm{~mA} / \mathrm{m}$ in the range $0.09 \mathrm{~m} \leq$ for all $z \leq 1.28 \mathrm{~m}$.

We can suppose that when we power the lower antenna the section covered by the field will be the mirror of the one covered by the upper: in this case the section covered will belong to the range $0.80 \mathrm{~m} \leq$ $z \leq 1.99 \mathrm{~m}$. Joining the two antennas we will cover all the $z$ in the range between $0.09 \mathrm{~m}$ and $1.99 \mathrm{~m}$. If we consider an $y-z$ plan at $x=-0.56 \mathrm{~m}$ and we power both the antennas at the distance of few milliseconds we can see that $\left|H_{x}\right|>60 \mathrm{~mA} / \mathrm{m}$ for all $z$ and for all $y$.

(ii) $\left|H_{y}\right|$ : the considerations on $\left|H_{y}\right|$ are notably easier because the structure covers all the operative volume with enough strength to read a tag, even reaching a distance up to $1.40 \mathrm{~m}$.

(iii) $\left|H_{z}\right|$ : on the $x-z$ plan for $y=1 \mathrm{~m}$ and $x=0.14 \mathrm{~m}$ we have that $\left|H_{z}\right| \geq 60 \mathrm{~mA} / \mathrm{m} 0.66 \mathrm{~m} \leq$ for all $z \leq$ $1.80 \mathrm{~m}$ and $0 \leq$ for all $z \leq 0.15 \mathrm{~m}$. In these intervals $\left|H_{z}\right| \geq 60 \mathrm{~mA} / \mathrm{m}$ for all $y \in(0,1 \mathrm{~m})$. 


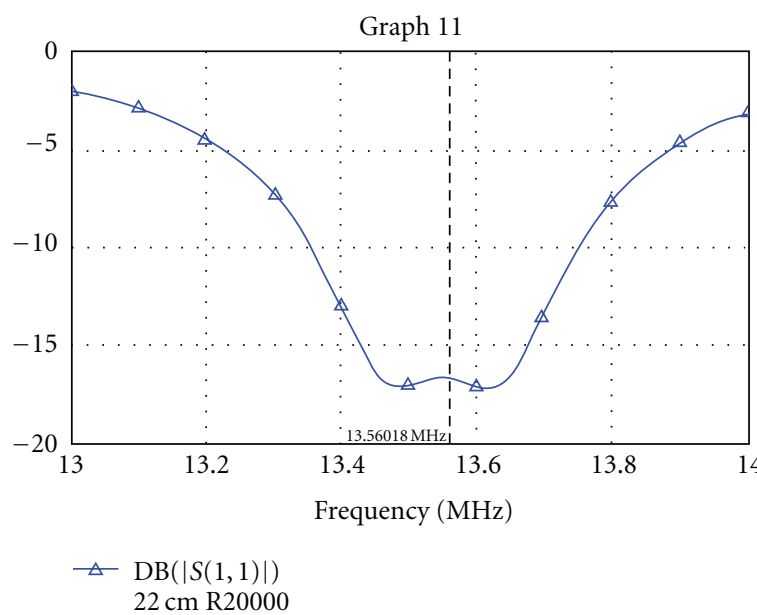

(a)

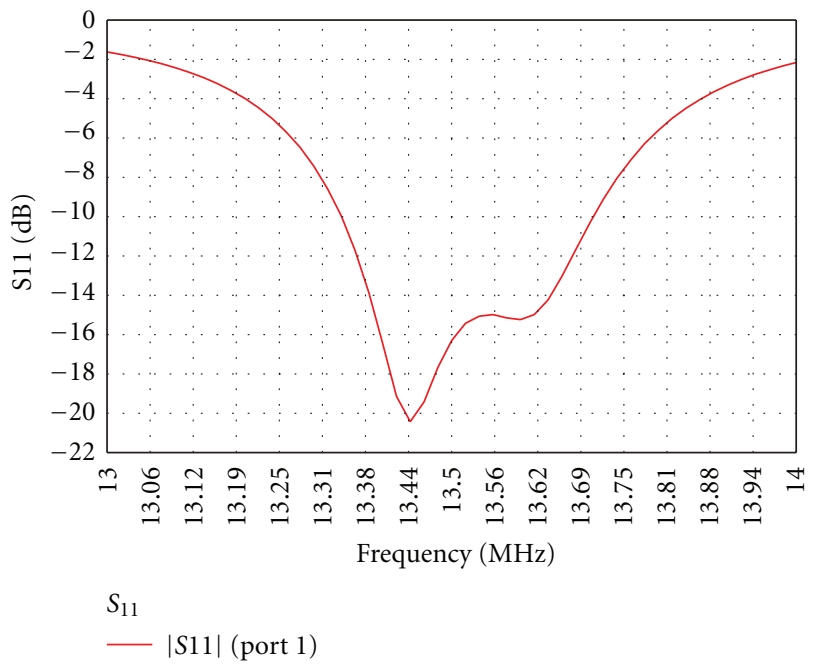

(c)

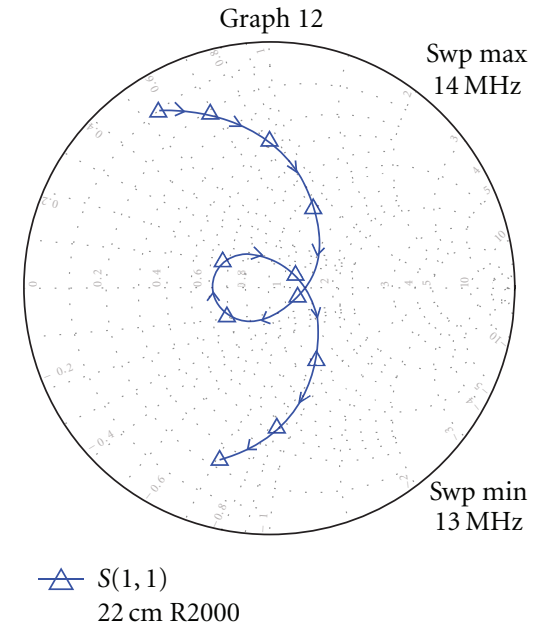

(b)

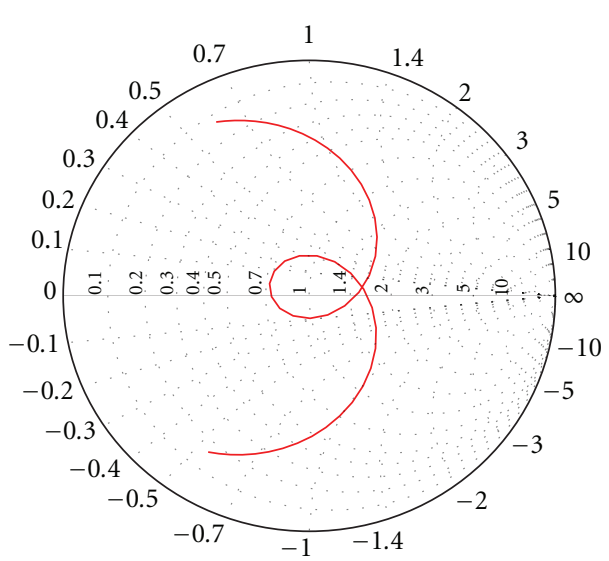

$S_{11}$ $-\left|S_{11}\right|($ port 1$)$

(d)

Figure 5: Reflection coefficient on the cartesian graphic and on the Smith Chart with AWR (a and b) and with POSTFEKO (c and d).

Finally, from the dimensions of the system we can assume that when we power the second antenna we have $\left|H_{z}\right| \geq$ $60 \mathrm{~mA} / \mathrm{m}$ for all $y \in(0,1 \mathrm{~m}), 0.22 \mathrm{~m} \leq$ for all $z \leq 1.36 \mathrm{~m}$ and $1.87 \leq$ for all $z \leq 2.02 \mathrm{~m}$, having then the volume belonging to half gate fully covered.

5.4. Varying the Antenna Factor $Q$. We saw that in the matching circuit of each antenna, simulated with AWR, we put the value of $R=20 \mathrm{~K} \Omega$; in reality we could also have reached the matching with lower values of $R$ : in particular we studied the cases with $R=15 \mathrm{~K} \Omega, R=10 \mathrm{~K} \Omega$ and $R=5 \mathrm{~K} \Omega$. Evidently, using these values also the values of the capacities vary, but it is important to underline that the antenna factor $Q$ decreases, due to the relation of direct proportionality between $Q$ and $R$.

In Figure 6 we can see $\left|S_{11}\right|_{\mathrm{dB}}$ in cartesian coordinates and on the Smith chart in relation to the studied cases: the red graph represents the $R=20 \mathrm{k} \Omega$ case, the blue graph represents the $R=15 \mathrm{k} \Omega$ case, the violet graph represents the $R=10 \mathrm{k} \Omega$ case, and the brown graph represents the $R=5 \mathrm{k} \Omega$ case. We can thus observe that the band at $-10 \mathrm{~dB}$ is obviously wider with a decrease of $R$, but we can see that with $R=5 \mathrm{~K} \Omega$, even if we have the widest band $(700 \mathrm{KHz})$, the phenomenon of the double peak does not appear, and the covering of the operative volume is definitely the worst.

We can then conclude that the double resonance is extremely useful because with the two peaks, in correspondence to the frequencies of the subcarriers, the band is wide enough to transmit and at the same time $Q$ is high enough to allow the existence of a sufficiently high irradiated magnetic field.

The analysis of the generated fields with POSTFEKO produced the following results.

(i) $R=5 \mathrm{~K} \Omega$ : considering the maximum plan for $x=$ $0.35 \mathrm{~m}$ and $0.12 \mathrm{~m} \leq$ for all $z \leq 1.90 \mathrm{~m},\left|H_{x}\right|>$ $60 \mathrm{~mA} / \mathrm{m}$ only up to $y=0.8 \mathrm{~m}$; considering the maximum plan for $x=0$ and $0.12 \mathrm{~m} \leq$ for all $z \leq 1.90 \mathrm{~m}$, 


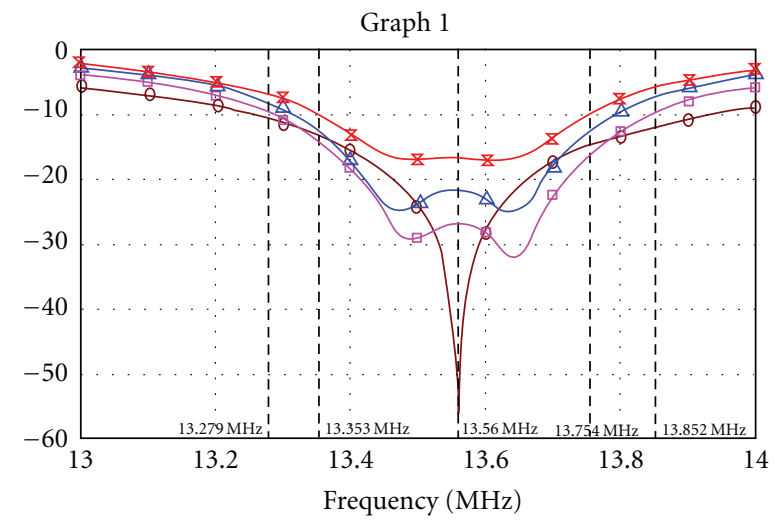

$\varangle \mathrm{DB}(|S(1,1)|) 22 \mathrm{~cm} \mathrm{R} 20000 \rightarrow \square-\mathrm{DB}(|S(1,1)|) 22 \mathrm{~cm} \mathrm{R} 10000$ $\triangle \mathrm{DB}(|S(1,1)|) 22 \mathrm{~cm} \mathrm{R} 15000 \rightarrow \mathrm{DB}(|S(1,1)|) 22 \mathrm{~cm} \mathrm{R} 5000$

(a)

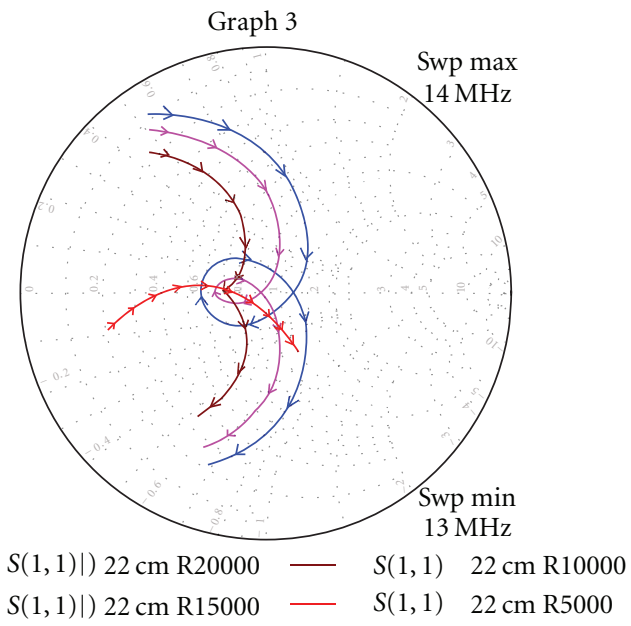

(b)

Figure 6: Reflection coefficient for different values of $R$ on the cartesian graphic (a) and on the Smith Chart (b).

$\left|H_{y}\right|>60 \mathrm{~mA} / \mathrm{m}$ up to $y=1.20 \mathrm{~m}$; considering the maximum plan for $x=0$ and $0.12 \mathrm{~m} \leq$ for all $z \leq$ $1.90 \mathrm{~m},\left|H_{z}\right|>60 \mathrm{~mA} / \mathrm{m}$ up to $y=0.8 \mathrm{~m}$.

(ii) $R=10 \mathrm{~K} \Omega$ : considering the plans described above, $\left|H_{x}\right|>60 \mathrm{~mA} / \mathrm{m}$ up to $y=0.8 \mathrm{~m} ;\left|H_{y}\right|>60 \mathrm{~mA} / \mathrm{m}$ up to $y=1.2 \mathrm{~m} ;\left|H_{z}\right|>60 \mathrm{~mA} / \mathrm{m}$ up to $y=0.8 \mathrm{~m}$.

(iii) $R=15 \mathrm{~K} \Omega$ : considering the plans described above, $\left|H_{x}\right|>60 \mathrm{~mA} / \mathrm{m}$ up to $y=0.9 \mathrm{~m} ;\left|H_{y}\right|>60 \mathrm{~mA} / \mathrm{m}$ up to $y=1.3 \mathrm{~m} ;\left|H_{z}\right|>60 \mathrm{~mA} / \mathrm{m}$ up to $y=0.9 \mathrm{~m}$.

We can see that in none of these case we can have a reading of the tags up to $1 \mathrm{~m}$ in every direction: in the first and in the second case we have a homogeneous reading up to $0.8 \mathrm{~m}$, while in the third we arrive at $0.9 \mathrm{~m}$.

5.5. Varying the Geometry of the Gate. Simulations have been made also with different lengths of the overlapping section. In particular we considered 3 different alternative overlapping lengths $(12 \mathrm{~cm}, 18 \mathrm{~cm}$, and $44 \mathrm{~cm})$ and the case with no overlapping (with a $2 \mathrm{~cm}$ distance between the antennas).

Figure 7 shows the reflection coefficient $\left|S_{11}\right|_{\mathrm{dB}}$ in the cases considered together with the final $22 \mathrm{~cm}$ solution (red graph in Figure 7).

In these four cases we had the following results.

(i) In the $12 \mathrm{~cm}$ case (Blue graph in Figure 7) we had a homogeneous reading up to $0.7 \mathrm{~cm}$

(ii) In the $18 \mathrm{~cm}$ case (Violet graph in Figure 7) we had a homogeneous reading up to $0.6 \mathrm{~cm}$.

(iii) In the $44 \mathrm{~cm}$ case (Brown graph in Figure 7) we had a homogeneous reading up to $0.6 \mathrm{~cm}$.

(iv) In the not overlapped case (Green graph in Figure 7) we had a homogeneous reading up to $0.7 \mathrm{~cm}$.

In none of these cases we reached satisfying results.

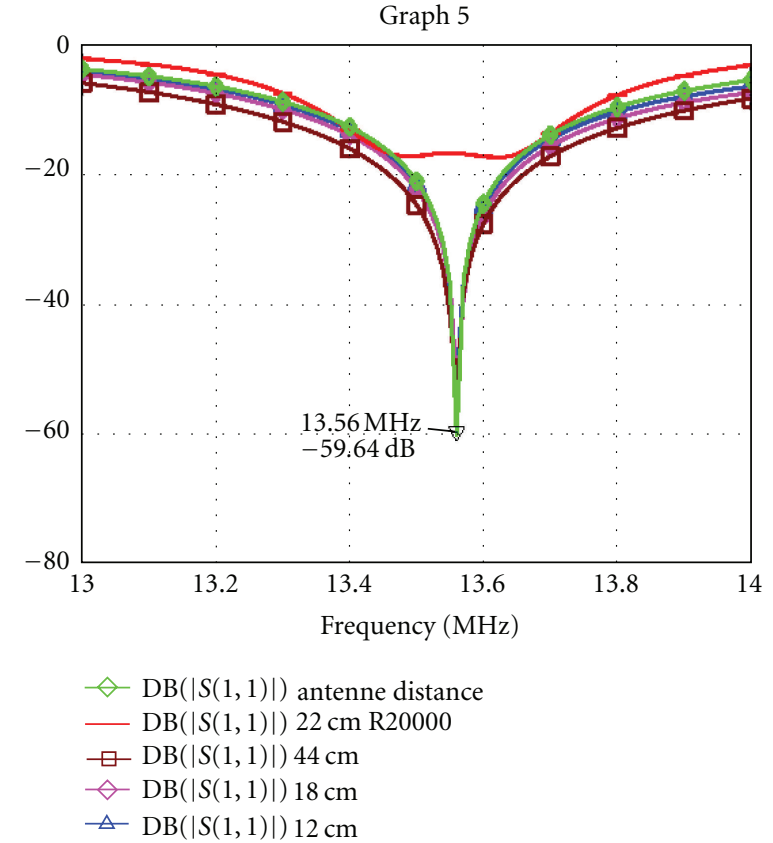

Figure 7: Reflection coefficient for different values of the overlapping surface on the cartesian graphic.

\section{Hardware Implementation}

Following the positive results of the simulations we went on with the realization of a prototype. The following materials were used:

(i) copper tubes for the antennas (16 mm diameter);

(ii) passive electronic elements (inductances, resistors, etc.) for the matching circuit;

(iii) wooden structures to hold the antennas. 


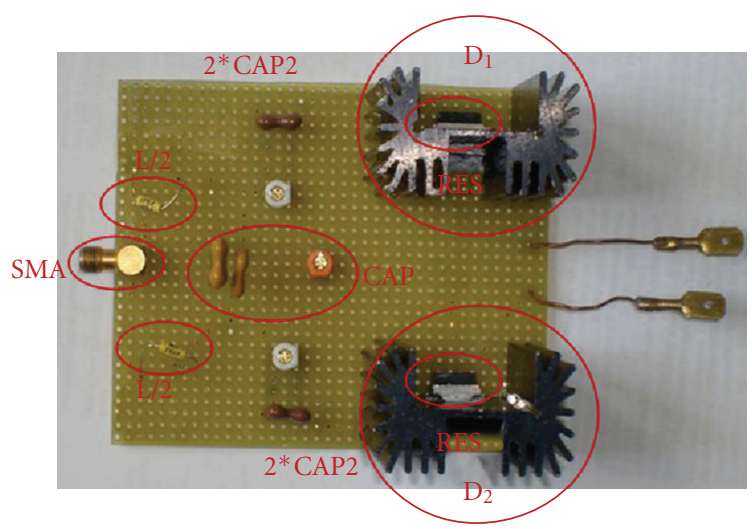

(a)

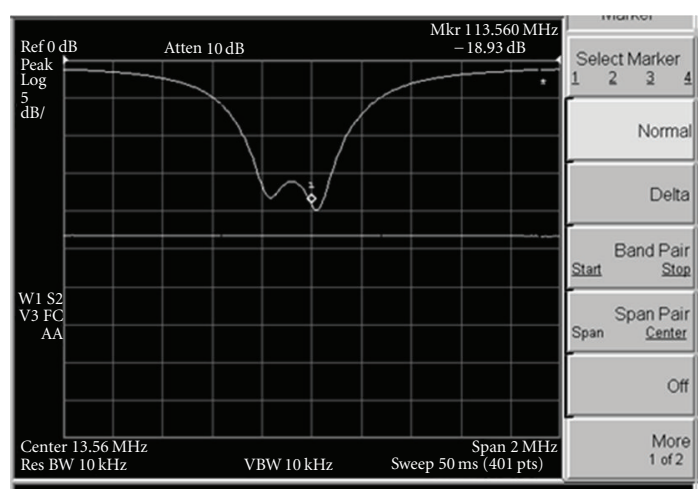

(b)

FIGURE 8: The real matching circuit (a) and the reflection coefficient as seen on the screen of the spectrum analyzer (b).

The wide diameter of the tubes has been necessary because simulations showed that we had better results for what concerned the strength and the shape of the magnetic field, while with a thinner tube the equivalent inductance of the antenna would have been higher (over $6 \mu$ Henry) making the matching circuit unstable due to the very low capacities required.

6.1. The Real Matching Circuit. After the realization of the antenna structure we went on with the implementation of the matching circuit for both the antennas, paying attention to use the same components with the same values, with the exception of the trimmers that have been regulated by hand during the matching phase.

The matching circuit realized for each antenna includes the components shown in Figure 8(a), where $L$, CAP, and CAP2 e RES correspond to the components seen in the simulated circuit while $D_{1}$ and $D_{2}$ are heat sinks. The lateral symmetric capacities (CAP2) and the central capacities (CAP) are formed by capacitors with constant capacity and capacitors with variable capacity (trimmers) in parallel. Once realized the circuit the following step focused on the matching of each antenna: this has been done linking, through a coaxial cable, the SMA connector of the circuit with a E4403B spectrum analyzer. In order to avoid the detuning phenomenon for the 4 antennas system the following prescriptions concerning the coaxial cables have been taken in account the following.

(i) To reduce the noise effects, a ferrite core has to be positioned, with at least three loops of the cable, at each end of the coaxial cable.

(ii) The coaxial cable linking the multiplexer and the reader has to be at most $50 \mathrm{~cm}$ long.

(iii) The coaxial cables connecting the antennas must have the same length $(\lambda / 2$ is the best choice to simplify the matching).

(iv) The matching has to be made separately for each half of the gate keeping in mind that while a cable is linked to the spectrum analyzer, the other cable must have an unlinked end and the other end connected to the second circuit, because this will be the configuration that the reader will find when the antennas will be powered one by one at a distance of few milliseconds.

Once the analyzer has been calibrated and connected the antenna, the trimmers have been regulated watching the waveform on the analyzer screen (Figure 8(b)), in order to obtain a matching similar to the one of the simulations. Initially the trimmers have been chosen with wide ranges of capacitive values $(6-50 \mathrm{pF})$. Subsequently, once found a good matching, they have been replaced with other trimmers providing lower range values (increasing also CAP and CAP2 if necessary). Once reached the final matching, it has been found that the band at $-10 \mathrm{~dB}$ was $680 \mathrm{kHz}$, then wider than the estimated one $(\sim 400 \mathrm{kHz})$. A following test has shown that some of transponders crossing the gate were not read: this means that the irradiated magnetic field was not strong enough to allow the identification of the tags in every orientation. This fact is probably due to capacitive phenomenons that make the equivalent inductance of the real antenna different from the one of the simulated antenna: as a consequence the antenna factor $Q$ is lower than the estimated one and then also the field strength is reduced. This fact also justifies the difference in the bandwidth.

For this reason it has been necessary to double the value of the resistances RES (from $20 \mathrm{~K} \Omega$ to $40 \mathrm{~K} \Omega$ ) in order to increase $Q$ and the emitted field strength, and to vary all the values of the capacities to obtain the identification of the tags on the whole crossing.

The final circuit for every antenna is implemented with the following components:

$$
\begin{gathered}
\mathrm{RES}=40 \mathrm{~K} \Omega, \\
\mathrm{CAP}=334-342 \mathrm{pF}, \\
2 \cdot \mathrm{CAP} 2=48.3-51.8 \mathrm{pF}, \\
L=360 \mathrm{nH} .
\end{gathered}
$$

The double resonance peak appears on the cartesian graph of the reflection coefficient provided by the spectrum analyzer, proving the fact that both the antennas are matched and 
resonant at two different, but very close frequencies: the mutual coupling phenomenon among the loops is used to widen the band using the two close resonances.

6.2. Measures of the Reading Distance. Once the matching of the system was achieved we moved to the test of the reading distances. The measures have been made using a FEIG ID ISC.LR2000 reader, ISO15693 compliant: its ID ISOStart configuration software has been used to test the functionalities of the reader, the reading range, and the communication with the tags. The measure of the maximum reading distance has been carried out using the Test-ISO Inventory procedure, provided with the control software, which performs a continuous scanning of the operative volume of the antenna reading, in case of detection, the serial number of the tag. The tags used for the measures are manufactured by Texas Instrument (I-Code 1 Tags), they are ISO15693 compliant and they present a $85 * 44 \mathrm{~mm}$ surface.

Studying only half gate (two antennas) we verified that a tag was correctly read in all the volume up to $1 \mathrm{~m}$ of distance. In particular for each orientation of the tag we found the following results:

(i) a tag, whose surface was oriented along the $y$ direction and crossing the gate along the $x$ direction was read at distance up to $1.10 \mathrm{~m}$;

(ii) a tag, whose surface was oriented along the $x$ direction and crossing the gate along the $x$ direction was read at distance up to $1.03 \mathrm{~m}$;

(iii) a tag, whose surface was oriented along the $z$ direction and crossing the gate along the $x$ direction was read at distance up to $1.00 \mathrm{~m}$.

This means that a tag is detectable in all the three orientation across a $2 \mathrm{~m}$ wide and $2 \mathrm{~m}$ high gate.

The testing of the system with all the four antennas has confirmed these results, leaving the matching parameters practically unchanged.

6.3. Interaction with Other Materials. Another set of tests has been performed in order to measure the probability of error in the reading of tags in presence of not electromagnetically transparent materials.

We saw that the impact on the transmission of metallic materials and polar liquids is obviously extremely harmful (reduction of $\simeq 60 \%$ in the first case and $\simeq 30 \%$ in the second) when the distance between the tag and these materials is less than $10 \mathrm{~cm}$. In contrast, with transparent materials (e.g., paper materials) the system works with excellent performances $(\simeq 100 \%)$. It has also been proved that the presence of two or more tags in the same area compromises the reading only when the tags are perfectly overlapped and their distance is lower than $4 \mathrm{~cm}$.

In particular the tests have been carried out positioning the tags on the following items and moving them across the gate in the middle of the crossing section (the worst case):

(i) empty paper packages;

(ii) plastic bottles filled with water; (iii) aluminium cans;

(iv) human body.

For what concerns non interfering materials (paper), the reading rate was around $100 \%$. In particular

(i) with 5 tags put randomly inside a paper box, performing 20 crossings of the gate the rate was $5 / 5$ for all the crossings;

(ii) with 8 tags put randomly, on 20 crossing we had the following rates: $8 / 8$ in 18 crossings and $7 / 8$ in 2 crossings;

(iii) with 8 tags, 5 of which oriented in the position of worst coupling, on 20 crossings we had the following rates: $6 / 8$ in 2 cases, $7 / 8$ in 12 cases and $8 / 8$ in 6 cases.

In the case of plastic bottles two tests were undertaken.

(i) 20 crossings were made with three bottles equipped with a transponder, put randomly inside a paper box: in this case the reading rate was always $3 / 3$.

(ii) Positioning the three bottles with the transponders in position of worst coupling the rate decreased at $2 / 3$ in all the 20 crossings.

In the case of metallic materials is evident that no reading is possible when the tag is in contact with metal. In particular, in the case of aluminum cans the transponder was identified with a $100 \%$ rate only when the distance from the can is higher than $10 \mathrm{~cm}$. Anyway, with a distance from 5 to $10 \mathrm{~cm}$ the results were still satisfying, with a rate around $60 \%$.

A final test was carried out to analyze the interaction with the human body. No problems (100\% successful readings) were noticed when the transponder was positioned inside a pocket of a man crossing the gate.

6.4. Law Requirements. Every time that a technological system is studied to be utilized in public environments, it has to be compliant with all the regulations concerning the safety of the final users. In the case of RFID systems European regulation is developed on two different levels:

(i) CEPT recommendations, whose decisions are not binding for the member countries;

(ii) European Commission directives, whose decisions are binding for the EU member countries and its lack of actuation is subject to the infraction procedure with the following economic sanctions.

In general these organizations set the operative band or frequency, the emitting power and the maximum time of communication between tags and readers. For what concerns the RF power, this term can generate confusion because there are some different methods of coupling between transponder and reader, depending on the operative frequency.

For frequencies up to $13.56 \mathrm{MHz}$ we have inductive coupling (the system operates in close field conditions) and we can speak of maximum field strength ( $\mathrm{H}$-field), expressed in $\mathrm{dB} \mu \mathrm{A} / \mathrm{m}$. The CEPT ERC/REC 70-03 recommendation 
determines the technical requirements for the use of Short Range Devices (SRD) for the CEPT countries [12].

The recommendation is divided in 13 annexes: number 9 and 11 are the ones concerning the RFID technology. Annex 9 covers the bands and regulates the parameters for the inductive applications, including, for example, animal identification, alarm systems, access control, and items tracking.

Key points of CEPT ERC/REC 70-03 a.9 are

(1) frequency: $13.553-13.567 \mathrm{MHz}$ for RFID and EAS (Electronic Article Surveillance) only;

(2) intensity of magnetic field: $60 \mathrm{~dB} \mu \mathrm{A} / \mathrm{m}$ at $10 \mathrm{~m}$;

(3) duty cycle: no Restriction;

(4) channel spacing: no spacing.

Annex 11 does not concern instead our case because it specifies the limitations for the other three RFID frequency bands. In conclusion, we have to comply with annex 9, in particular with the second point concerning the maximum magnetic field strength.

6.5. The Project and the Laws. The first step towards the check of the requirements has been made once again with the simulations: we saw that the maximum value of magnetic field strength at $10 \mathrm{~m}$ of distance is $9.421 \cdot 10^{-4} \mathrm{~A} / \mathrm{m}$. The requirements recommend a $60 \mathrm{~dB} \mu \mathrm{A} / \mathrm{m}$ magnetic field strength at $10 \mathrm{~m}$ distance. $60=20 \lg _{10} x \Rightarrow x=10^{3}$ so this value can be expressed in linear measurement as $10^{3} \mu \mathrm{A} / \mathrm{m}=$ $10^{-3} \mathrm{~A} / \mathrm{m}$ : therefore the values of the simulations are under this threshold.

The second phase of the requirements check has been realized directly in laboratory on the final structure: the system has been powered with $8 \mathrm{~W}$ and the magnetic field has been measured with a probe linked to a spectrum analyzer. The obtained values have confirmed the positive results of the simulations.

\section{Conclusions}

In this paper we have shown how to realize an RFID gate detecting tags in every orientation across a $2 \mathrm{~m}$ wide and $2.15 \mathrm{~m}$ high section. This solution has been achieved through numerical simulations of the structure, through which the ideal geometry of the system and the adapted configuration of the matching circuit as been identified.

Following the simulations, a prototype of the structure has been realized and tested in laboratory: while some parameters (mainly for what concerns the matching circuit) required some adjustments, the results of the simulations were substantially confirmed. Tests concerning the performances of the system have involved the reading timing, the interaction with critical materials, the reading of overlapped transponders, and the total number of transponders readable simultaneously. All these tests have provided positive results, encouraging the prosecution of the research activities.

Additional work is going on in order to realize a structure to be adapted in a better way to the real destination environments. In particular future work is mainly focused on the realization of a system with reduced dimensions, in order to employ it in the highest number of possible different scenarios.

\section{References}

[1] K. Finkenzeller, RFID Handbook: Fundamentals and Applications in Contactless Smart Cards, Radio Frequency Identification and Near-Field Communication, John Wiley and Sons, 3rd edition, 2010.

[2] D. E. Brown, RFID Implementation, McGraw-Hill Professional, 2007.

[3] A. Goulbourne, "HF antenna design notes, technical application report," Tech. Rep. 11-08-26-003, Texas Instruments, Radio Frequency Identification Systems, 2003.

[4] C. A. Balanis, Antenna Theory. Analysis and Design, Harper \& Row, 1982.

[5] H. Lehpamer, RFID Design Principles., Artech House, Norwood, Mass, USA, 2008.

[6] S. Kawdungta, C. Phongcharoenpanich, and D. Torrungrueng, "Design of flat spiral rectangular loop gate antenna for HFRFID systems," in Proceedings of the Asia Pacific Microwave Conference (APMC '08), Hong Kong, December 2008.

[7] N. C. Karmakar, Handbook of Smart Antennas for RFID Systems, John Wiley and Sons, 2010.

[8] J. M. Rathod and Y. P. Kosta, "Low cost development of RFID antenna," in Proceedings of the Asia Pacific Microwave Conference (APMC '09), pp. 1060-1063, Singapore, December 2009.

[9] C. Reinhold, P. Scholz, W. John, and U. Hilleringman, "Efficient antenna design of inductive coupled RFID-systems with high power demand," Journal of Communications, vol. 2, no. 6, 2007.

[10] B. Jiang, J. R. Smith, M. Philipose, S. Roy, K. Sundara-Rajan, and A. V. Mamishev, "Energy scavenging for inductively coupled passive RFID systems," in Proceedings of the IEEE Instrumentation and Measurement Technology Conference, vol. 10, pp. 984-989, Ontario, Canada, May 2005.

[11] C. M. Zierhofer and E. S. Hochmair, "Geometric approach for coupling enhancement of magnetically coupled coils," IEEE Transactions on Biomedical Engineering, vol. 43, no. 7, pp. 708$714,1996$.

[12] European Standard (Telecommunications series). ETSI EN 300330, Part 1: technical characteristic and test methods, 1999. 

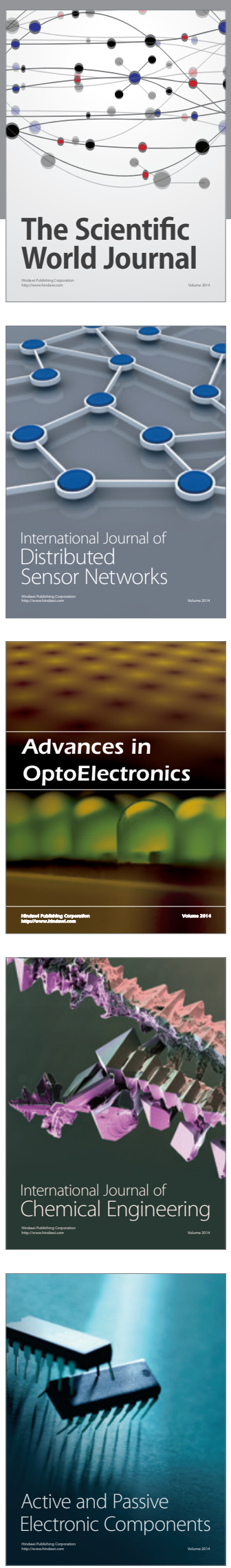
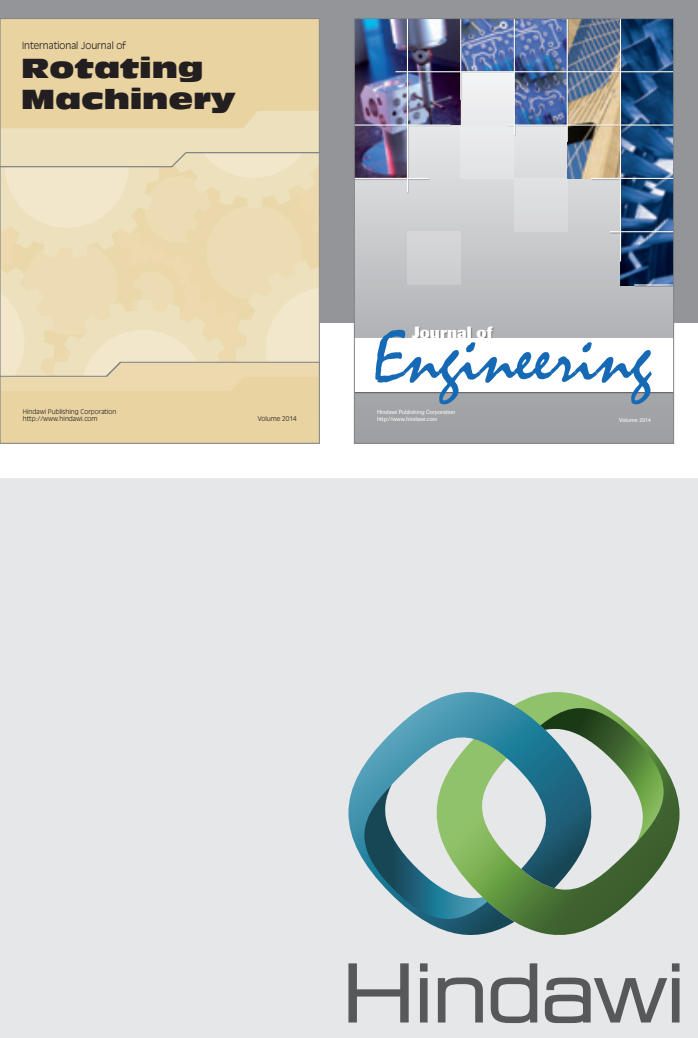

Submit your manuscripts at

http://www.hindawi.com
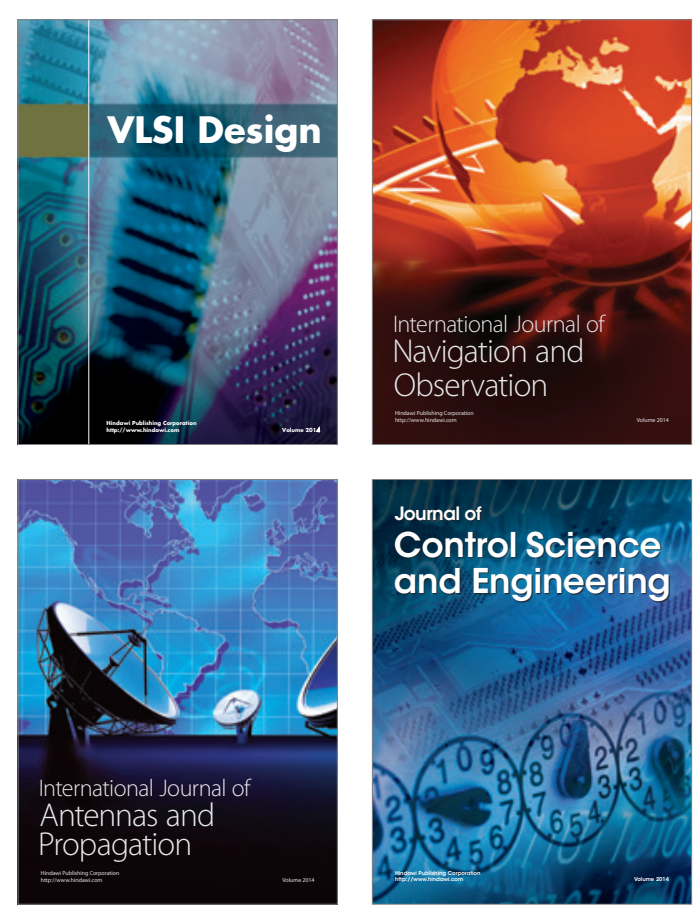
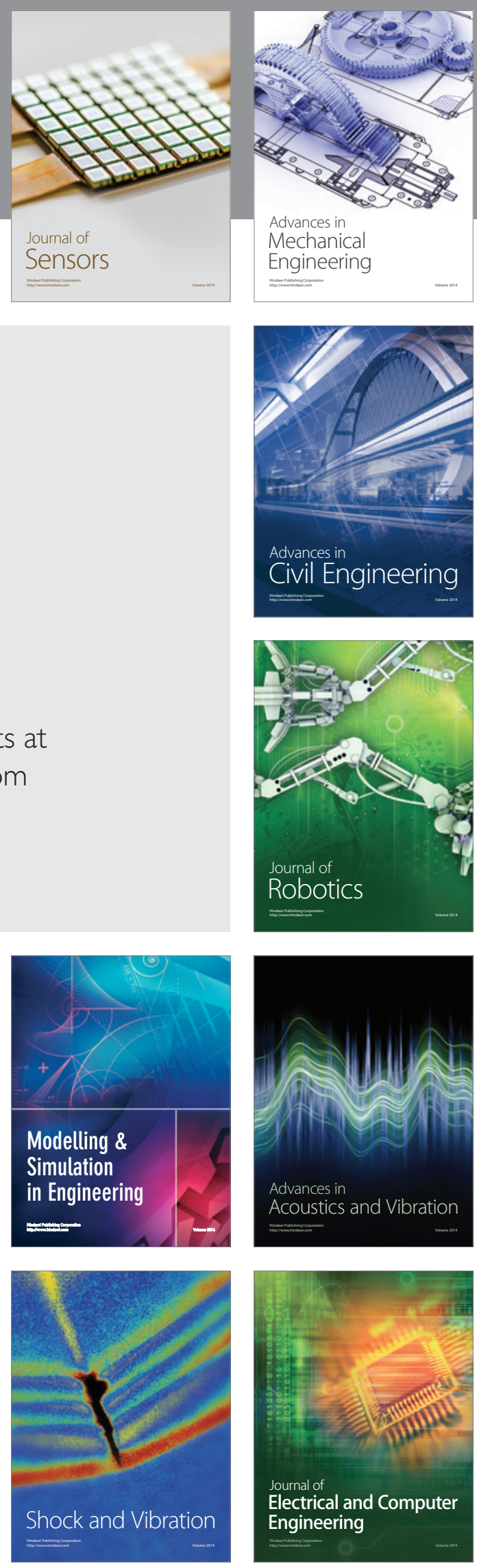